\title{
Waveform-preserving processing flow of multichannel seismic reflection data for ocean thermohaline structure reconstruction (revisited)
}

\author{
Josafat Ribeiro Leal Filho, Francisco Carlos Lajús. Junior, Antonio Henrique da Fontoura Klein, Stephan Paul
}

Universidade Federal de Santa Catarina - UFSC

\begin{abstract}
Copyright 2021, SBGf - Sociedade Brasileira de Geofísica.
This paper was prepared for presentation at the $17^{\text {th }}$ International Congress of the Brazilian Geophysical Society, held in Rio de Janeiro, Brazil, 08 November to 11 November, 2021.

Contents of this paper were reviewed by the Technical Committee of the $17^{\text {th }}$ International Congress of The Brazilian Geophysical Society and do not necessarily represent any position of the SBGf, its officers or members. Electronic reproduction or storage of any part of this paper for commercial purposes without the written consent of The Brazilian Geophysical Society is prohibited.
\end{abstract}

\section{Abstract}

Along the advent of 4D seismic exploration, the need to properly compensate spatio-temporal variations in the water column has become of utterly importance for the adequate characterization of reservoirs changes during their exploration phase. In this study we revisit a previously suggested processing flow that is focused on the recovery of oceanographic coherent structures (DAGNINO et al., 2017), and propose some modifications. The main reason for this is our restriction to work with already processed (by 3rd parties) data, that was mostly guided to proper characterization of sub-sea floor geologic structures. We found that our re-processing flow is able to recover signatures of oceanic thermohaline structures, thus having a potential for further inversion focused on detailed water layer velocity reconstruction.

\section{Introduction}

Reservoir management, including reservoir monitoring and surveillance, is a core part of oilfield development strategies and carbon dioxide storage projects. It provides industry experts with methods to optimize and track hydrocarbon recovery rates, or to supervise $\mathrm{CO} 2$ storage capacity. An ideal reservoir management tool should be able to observe and track the internal fluid movement, estimate the effect of its displacement, locate and predict future distributions of fluid (SAMBO et al., 2020). In this context, 4D seismography is a complementary tool for reservoir management (LUMLEY, 2001), especially to obtain measures of the speed of sound in the water column.

The most common methods include: picking zerooffset traveltime to the seabed, measurements of the travel time change of the sub-sea reflector with offset, and conventional velocity analysis to obtain averaged velocities in the water column (BRIGHT et al., 2015). However, a somewhat new approach, focused on highresolution estimation of the sound velocity in the water column considers the processing of ocean thermohaline reflections, generated by the impedance contrasts that occur within the water column (BRIGHT et al., 2015).

Regarding the particular care required to process such small thermohaline reflections, (DAGNINO et al., 2017) proposed a wave-form preserving workflow to filter and separately process parts of the seismic data in order to recover highly detailed termohaline structures. Figure 1 displays a general scheme of this workflow, which can be divided into four main blocks: 1) Noise attenuation with Butterworth filter and padding, 2) SVD, after linear (normalized) move-out correction, separating direct wave and reflections, 3) Direct wave treatment, with trace flagging and amplitude control, and 4) Treatment of the reflections, with amplitude control, normal move-out correction, dip filter and trace flagging.

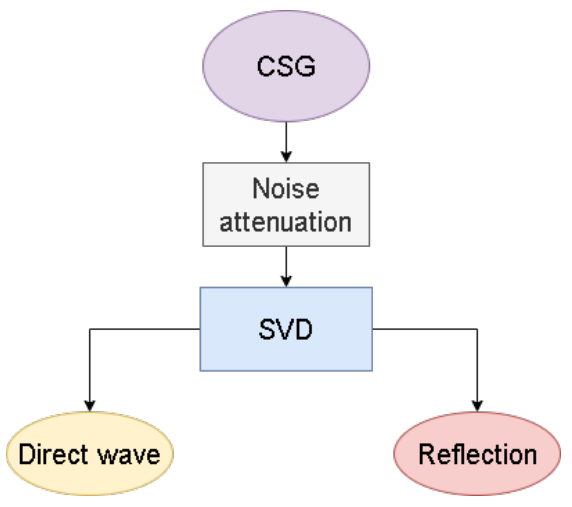

Figure 1: General workflow of (DAGNINO et al., 2017)

Although the main idea behind this workflow can be maintained, differences in the results are expected when input data is used that has been already processed for reservoir exploration. In this preprocessed data some artifacts in the portion of the data that represent the water column require additional re-processing to recover the oceanic structure's signature.

The complete list of the processing applied by a third party to our input data is as follows:
- Reformat;

- Gridded binning;

- Bad SP and traces flag.;

- Noise attenuation;

- Debubble;

- Residual denoise;

- Shot chan. amp. cor.;

- Deghosting (WiBand);
- Zero-Phasing;

- Residual Debbuble;

- Residual Denoise;

- Tidal \& WCS;

- Tzero;

- 3D SRME;

- 2D Radon;
- RMC 
Thus, in this work we present some additional processing steps adopted to remove either random noises and processing artifacts, of a previously-processed marine seismic acquisition.

\section{The processing sequence}

\section{Noise attenuation}

Analyzing a common shot family in our provided data, three processing artifacts are readily discernible: I) Inclined lines; II) High amplitude vertical noises; and III) Low-frequency hyperbolic noise. The first one are coherent noises in the Common Shot Gather (CSG), but appear randomly in some other gather choice, like the Common Offset Gather (COG). Therefore, FX-decon and trace-mix appear as candidate tools for removing these random noises, playing with their altered application in CS and CO gathers.
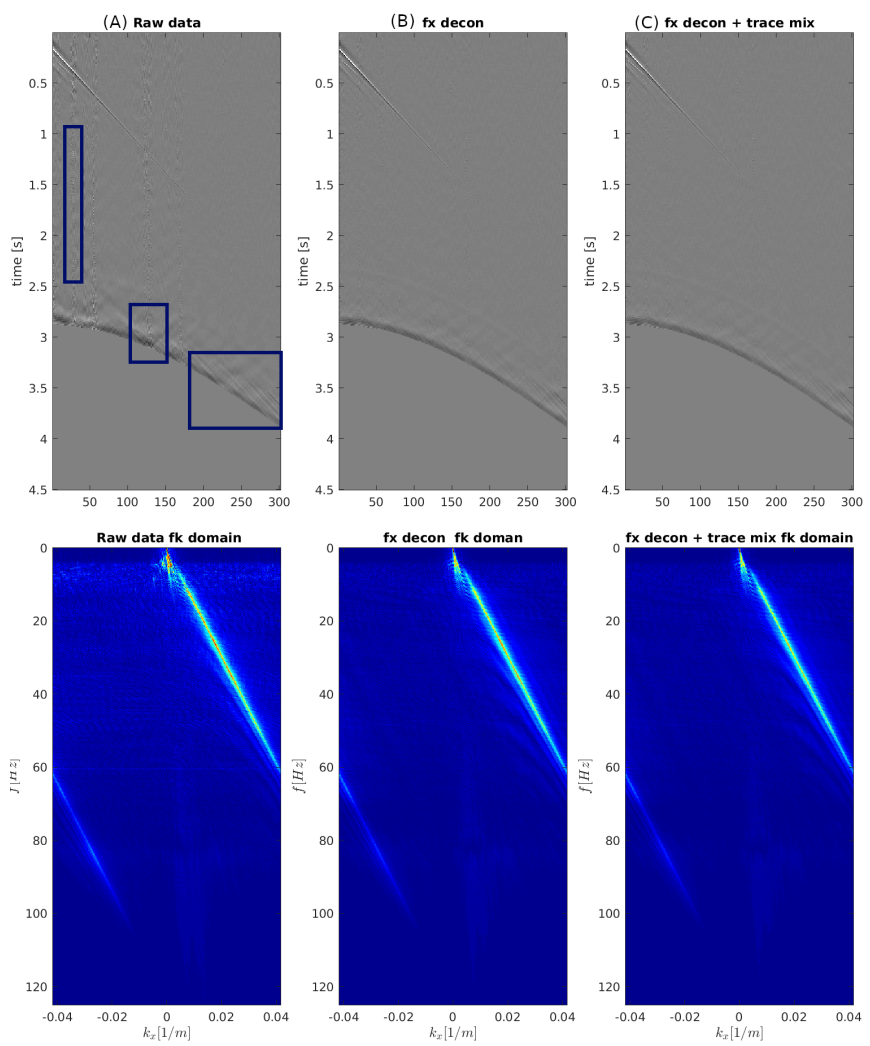

Figure 2: Gray image of field CSG : raw data, after fx-decon and after $\mathrm{fx}$-decon and trace mixing. Lower panels of $\mathrm{f}-\mathrm{k}$ transform, that are applied to monitor changes on the signal amplitude.

Figure 2 shows a comparison between raw data and gradually filtered data, in CSG. In the left upper panel residuals attributed to swell noise and processing noise, highlighted in blue, are clearly identifiable due to its high amplitudes. FX-decon is able to attenuate the noise highlighted in the upper left panel, mainly at low frequencies and close to the ocean floor as can be seen in the upper center panel. Additional trace mixing is able to remove residual noise, which is shown in the associated

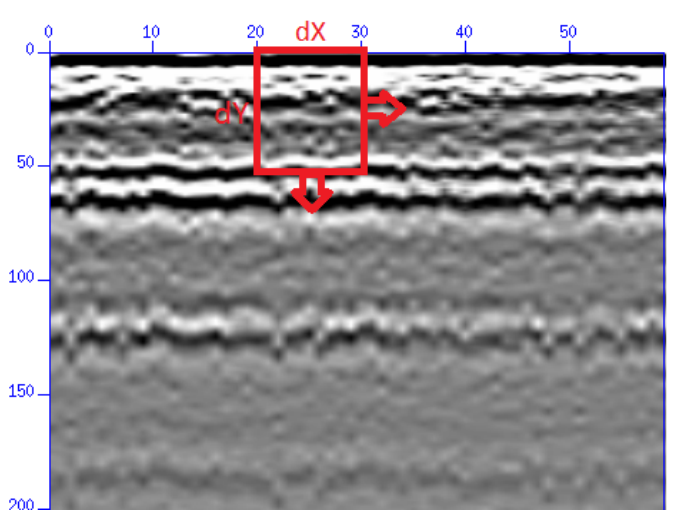

Figure 3: Example of an adaptive SVD movable window (LEAL, 2019)

upper right panel. Lower panels are correspondent f$\mathrm{k}$ transforms of the upper images, which allows detailed amplitude monitoring of the processed signal.

\section{Singular value decomposition}

For this step, adaptive SVD is used (PORSANI et al., 2013), which consists of applying conventional SVD in a small movable fixed size window (for instance, a $7 \mathrm{x}$ 7 window), horizontally and vertically along a normalized linear move-out adjustment of the seismic data. This is schematically shown in Figure 3. Results of this processing generates decomposed eigen-images (Els), that are optimally rank by the small window singular values, which are affected by the spatial and temporal alignments found in the neighbors of the window centered region.

The purpose of processing at this stage is mostly to remove residual noise, through a separation of optimally ranked aligned events, that are flattened by the normalized linear move-out (LMO) corrections applied to the direct wave. The significance of applying a LMO correction and normalization is exemplified in Figure 4. In this case, the bubble effect and other linear events that have a similar tendency as the direct wave are captured in the first El, followed by eventual thermohaline reflections and less spatially coherent fluctuations into the remainder $n-1$ Els. Normalization has the capacity of smoothing out the amplitudes for the most part of the direct wave, improving the decomposition into eigen-images in this region as seen in Figure $4(E)$.

Figure 5 starts with noise attenuated (FX-decon + trace mix - upper left panel) CSG, and displays resultant Els decomposition obtained via subsequent adaptative SVD application. The cumulative norm $\left(\left|\sum_{i=1}^{j} E I_{i}\right| /\left|\sum_{i=1}^{n} E I_{i}\right|\right)$ of the Els' amplitudes is shown in Figure 6 . It is seen that the $1^{s t} \mathrm{El}$ accounts for $\approx 83 \%$ of the total signal amplitude. Thus, the remaining Els contain the expected information of thermohaline reflections. Els 2 up to 5 account to something close to $16 \%$ of the total image amplitude, while Els 6 to 7 correspond to less than 1\%, being mainly composed of less coherent amplitudes.

Figure 7 shows the gradual formation of field CSG obtained by the increased sums of Els obtained just after adaptive SVD. Increased spatial detail is verified in the observed thermohaline reflections. 

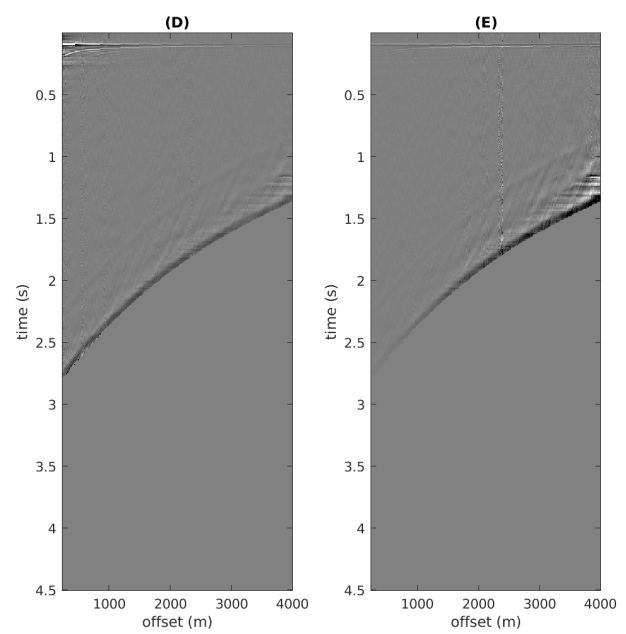

Figure 4: Gray image of field CSG : (D) after LMO, (E) after LMO and normalization
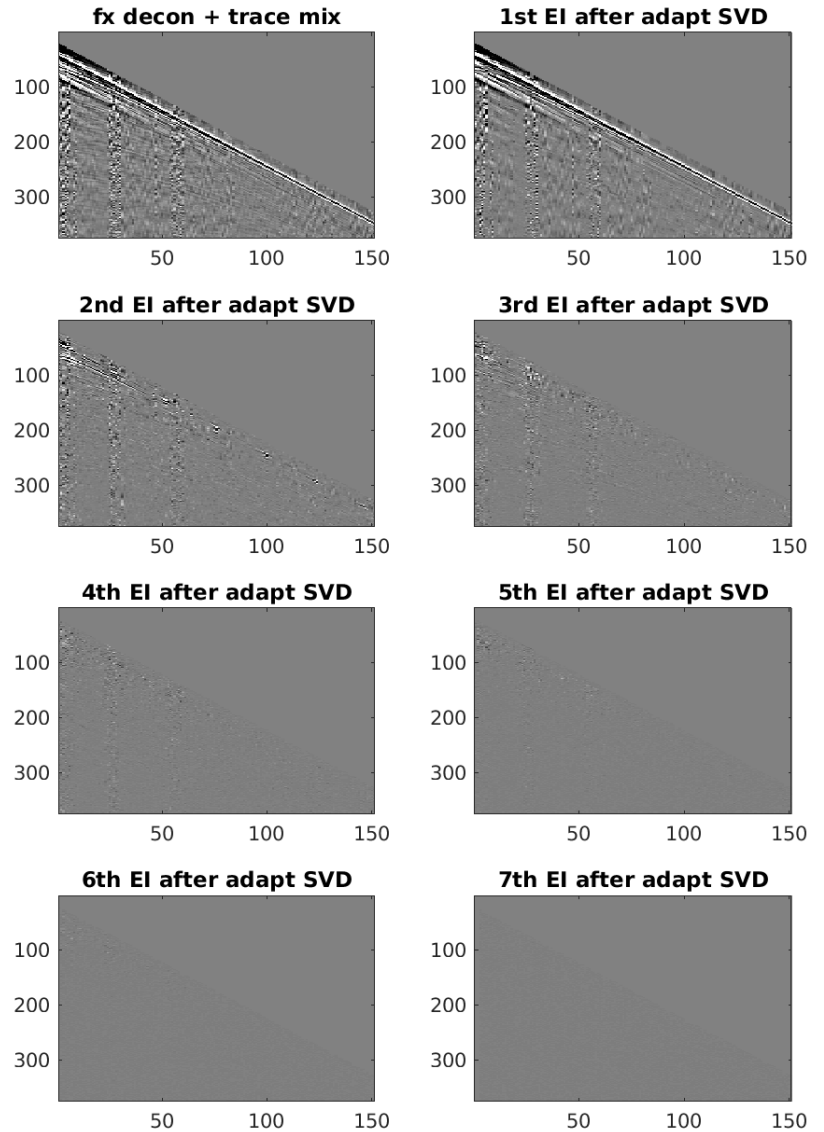

Figure 5: Gray images of field CSG after fx-decon and trace mix (upper left panel) and individual Els obtained via subsequent adaptative SVD

\section{Processing of reflection data}

After direct wave separation, subsequent processing is focused to increase even more the spatial and temporal coherence of the thermohaline reflections and to remove

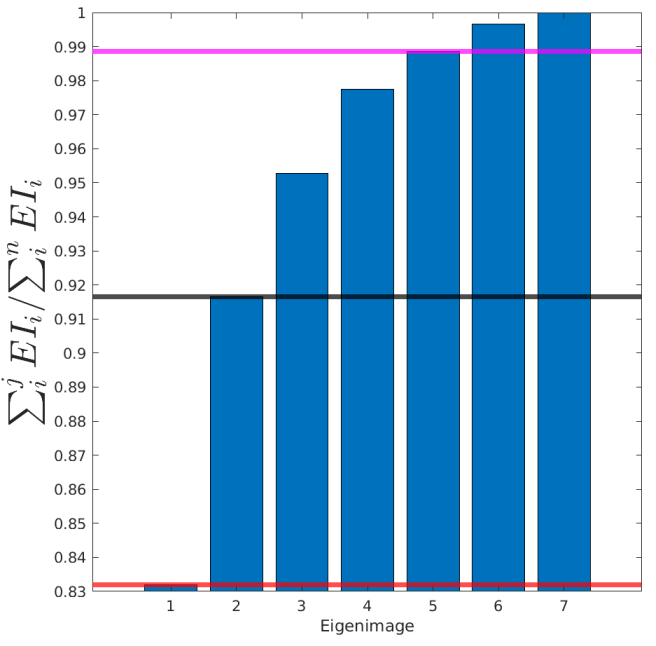

Figure 6: Cumulative norm of the Els
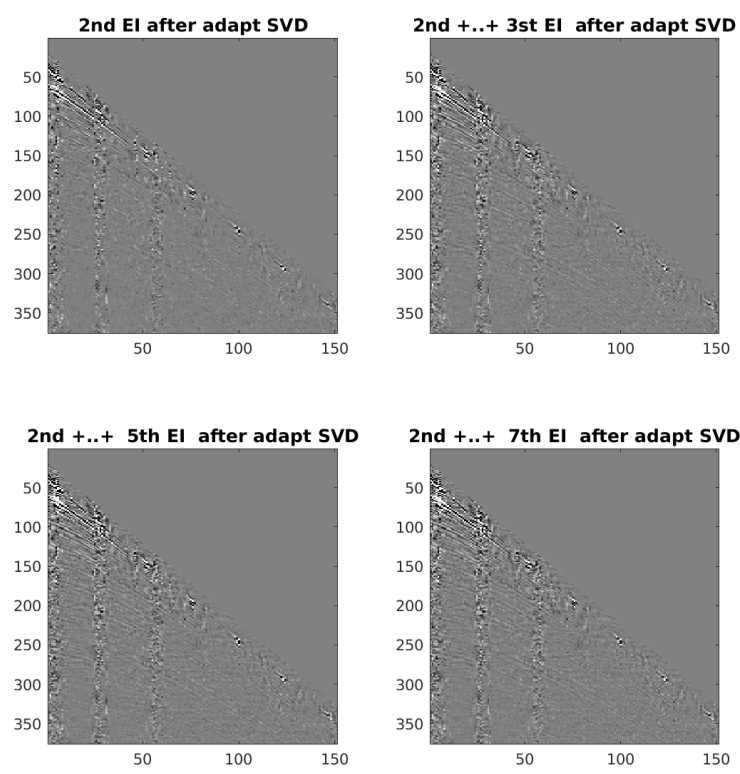

Figure 7: Gray image of field CSG created by the sum of eigenimages obtained by adaptive SVG

residual noise. This can be achieved by $\mathrm{fk}$ domain filtering, designed to maintain the region of thermohaline reflections, while mute is enforced elsewhere - on negative wavenumbers and/or slopes that are not consistent with the expected reflections. The impact of this fk filtering in the final image is shown in Figure 8 . The verified small contribution of the 6th and 7th Els, here verified in more detail to the final image, indeed justifies to further disregard of their contribution.

Comparisons of Figure 8 and Figure 9, exemplify how the cumulative sum of the 2nd up to the 5th El improves spatial and temporal resolution in the CSG, resulting in a amplitude gain mainly at frequencies above $60 \mathrm{~Hz}$. Stacked sections of the raw data (Figure 10) and the final data (Figure 11), further exemplify the total image gain for 

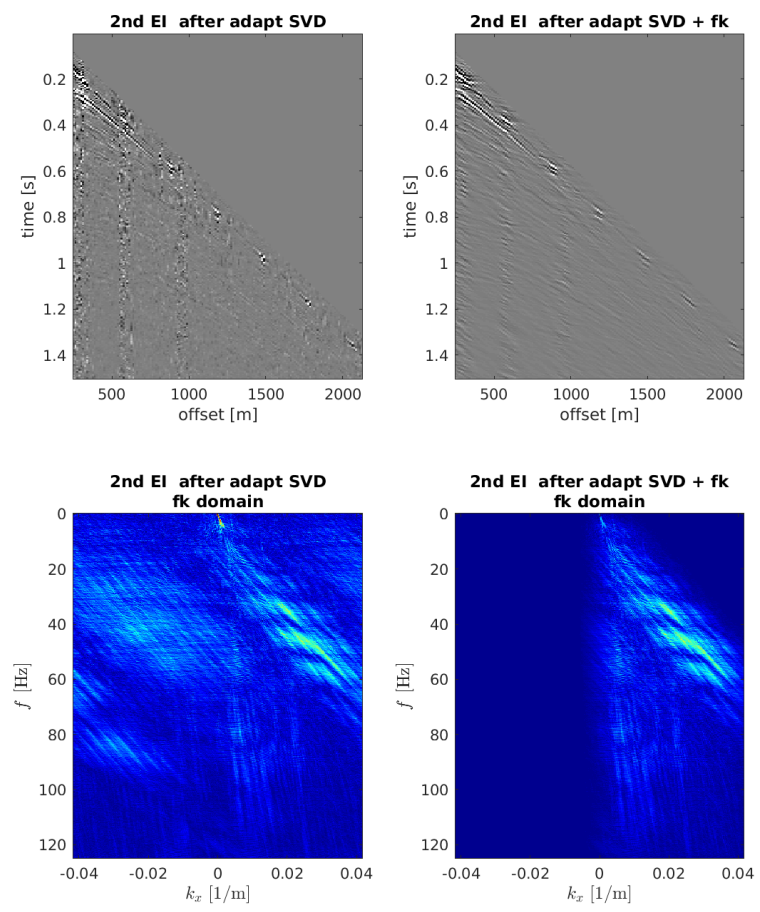

Figure 8: Results of adaptive SVD before fk filtering (left panels) and after fk filtering (right panels) for the 2nd El. Upper panels: CSG, lower panels: fk domain.

the proposed processing sequence focused on emphasize water layer reflections. Random and some of the coherent high amplitude noises (physical and processing-artifacts) were removed, allowing some visualizaton of thermohaline reflection signature, especially in the upper portion of the water column.

\section{Conclusions}

The additions presented in this paper to DAGNINO et al. (2017)'s workflow were found to fairly recover the signature of thermohaline reflections, even in the presence of a heavily previously-processed (by third parties) marine seismic data. The further combination of such processing flow for velocity inversion are currently being tested.

\section{Acknowledgements}

The authors are grateful to Carlos Theodoro and Gilberto Lima for suggesting application of FX-decon and tracemix, and to André Bulcão, Bruno Dias for much of the ongoing discussion regarding data preparation for seismic inversion. This research was supported by Petrobras through "Determinação da velocidade do som da coluna d'àgua utilizando dados de levantamentos sísmicos" project with Universidade Federal of Santa Catarina (UFSC), and ANP through the R\&D levy regulation, process: $2017 / 00071-6$.

\section{References}

BRIGHT, D.; JONES, C; and SELVAGE, J, 2015, Solving water column statics with seismic oceanography: 77th
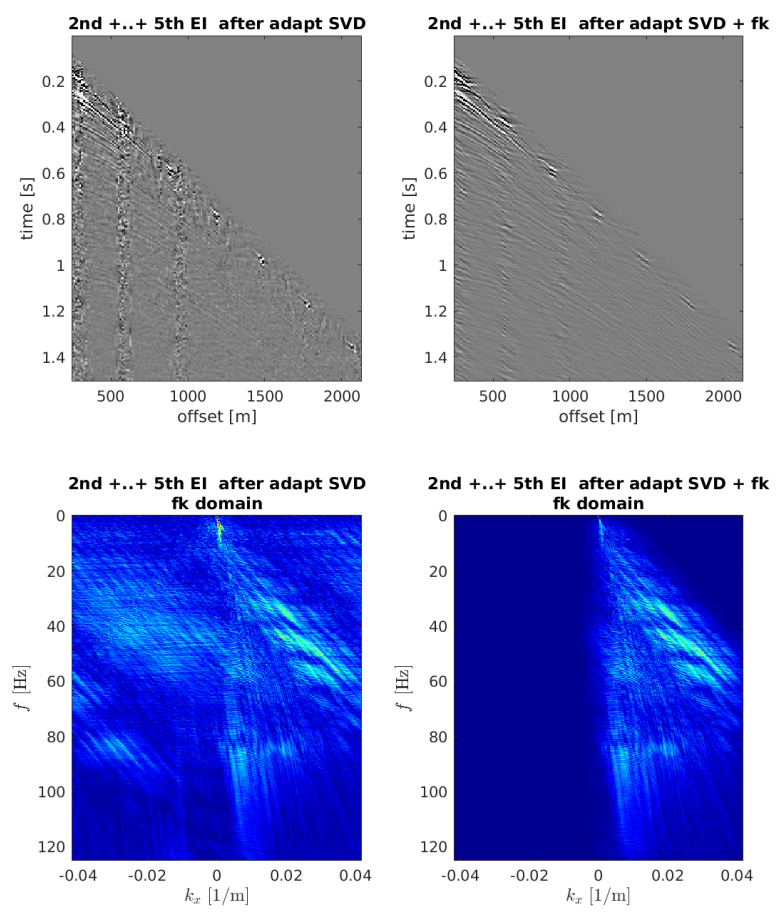

Figure 9: Result of adaptive SVD before fk filtering (left panels) and after fk filtering (right panels). Upper panels: CSG, lower panels: fk domain.

EAGE Conference and Exhibition 2015, 1-5.

DAGNINO, D.; SALLARÈS, V.; and RANERO, C., 2017, Waveform-preserving processing flow of multichannel seismic reflection data for adjoint-state full-waveform inversion of ocean thermohaline structure: IEEE Transactions on Geoscience and Remote Sensing, 56, 1615-1625.

LEAL, R., J. F., 2019, Oceanografia sísmica: Processamento de dados sísmicos de baixa frequência para mapeamento de estruturas termohalinas. (Monografia (Bacharel em Geofísica), UFBA (Universidade Federal da Bahia), Salvador, Brazil).

LUMLEY, D., 2001, Time-lapse seismic reservoir monitoring: Geophysics, 66, 50-53.

PORSANI, M.; URSIN, B; SILVA, M; and MELO, P, 2013, Dip-adaptive singular-value decomposition filtering for seismic reflection enhancement: Geophysical Prospecting, 61, 42-52.

SAMBO, C.; IFEROBI, C.; BABASAFARI, A.; S, R.; and AKANNI, O., 2020, The role of 4d time lapse seismic technology as reservoir monitoring and surveillance tool: A comprehensive review: Journal of Natural Gas Science and Engineering, 103312. 


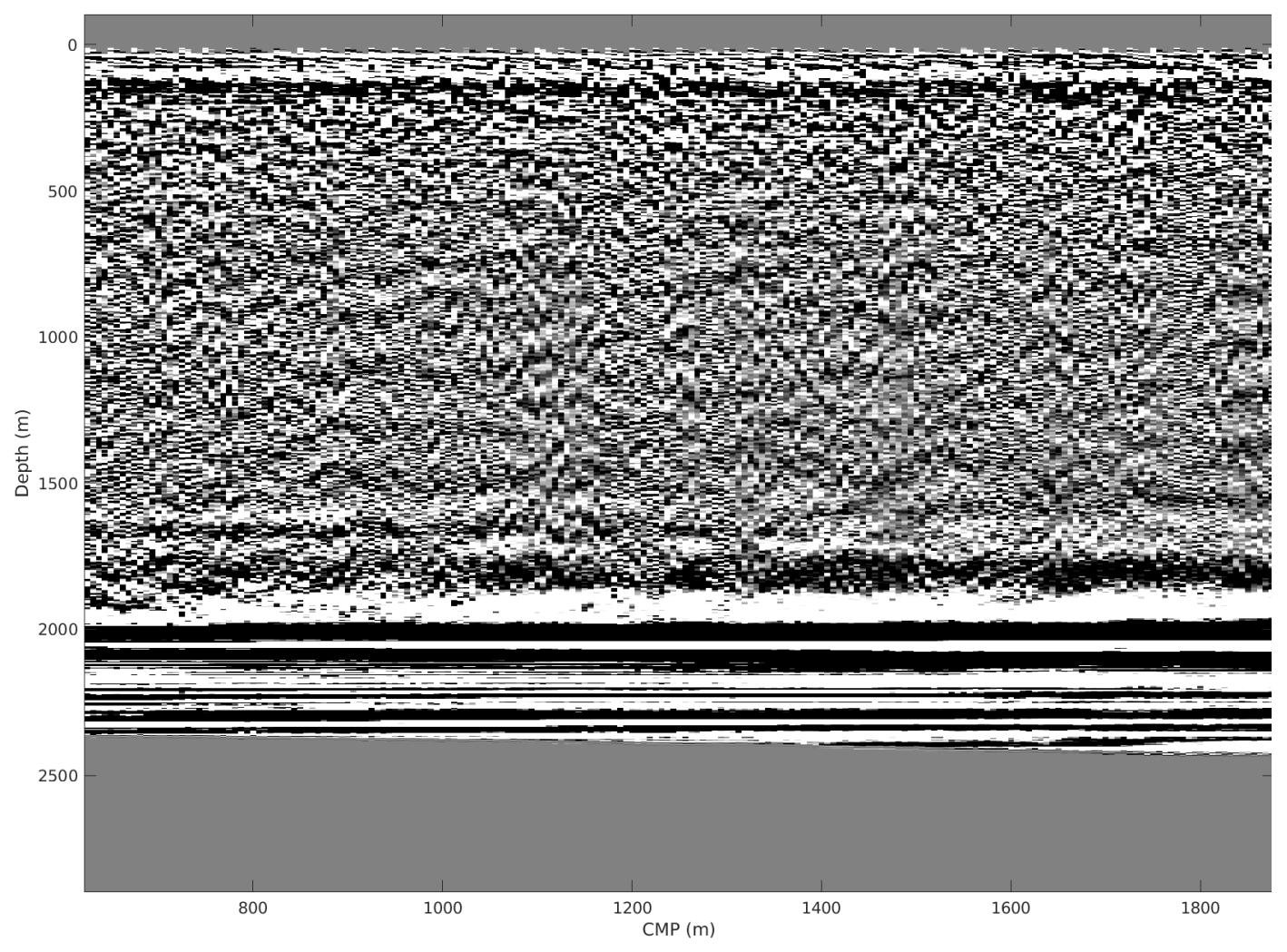

Figure 10: Stacked raw data

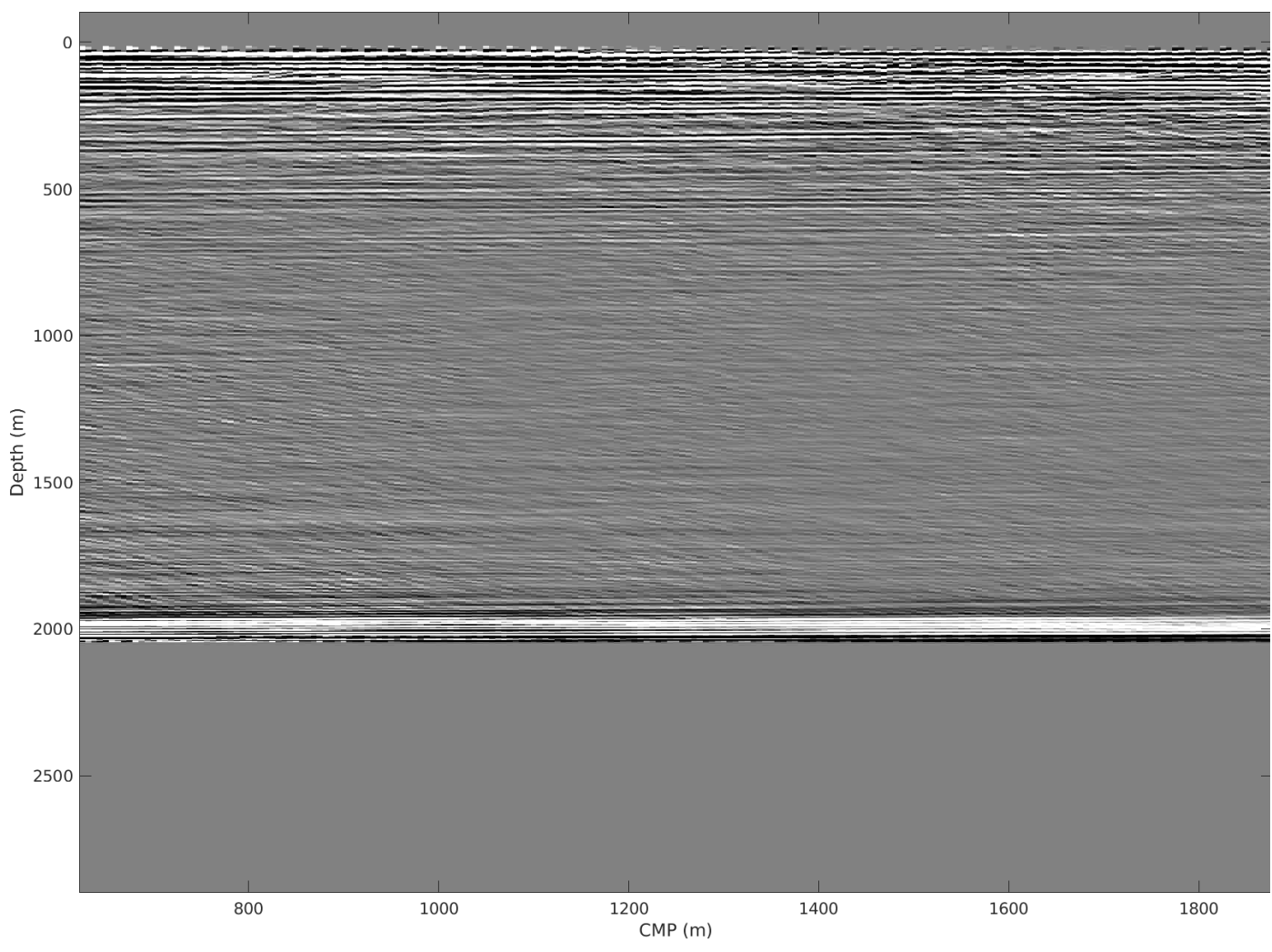

Figure 11: Stacked data after processing 